\title{
Small Intestinal Kaposi Sarcoma
}

National Cancer Institute

\section{Source}

National Cancer Institute. Small Intestinal Kaposi Sarcoma. NCI Thesaurus. Code C96059.

A Kaposi sarcoma that arises from the small intestine. 